\title{
miR-I45-5p Regulates the Proliferation, Migration and Invasion in Cervical Carcinoma by Targeting KLF5
}

This article was published in the following Dove Press journal: OncoTargets and Therapy

\section{Hui Cao \\ Guihua Pan \\ Shiqiang Tang \\ Ni Zhong \\ Huake Liu \\ Haizhi Zhou \\ Qin Peng \\ Yongbin Zou}

Department of Oncology, Chenzhou First People's Hospital, Chenzhou, People's Republic of China
Correspondence: Yongbin Zou Department of Oncology, Chenzhou First People's Hospital, 102 Luojiajing Road, Chenzhou 423000, People's Republic of China

Tel +86-13507357355

Email gjnzhu888@I63.com
Objective: Cervical carcinoma (CC) is a serious threat to women's health and few effective therapeutic methods have been discovered. The purpose of this study is to explore the underlying mechanism of miR-145-5p in CC.

Methods: Bioinformatics methods were employed to analyze the gene expression data of CC from TCGA database. qRT-PCR was used to detect the expression of miR-145-5p and KLF5 in CC cells, and Western blot was employed for the examination of KLF5 protein level. The targeted relationship between miR-145-5p and KLF5 was verified by a dualluciferase reporter assay. Moreover, CCK-8, wound healing assay and transwell invasion assay were used to analyze the effects of miR-145-5p overexpression or KLF5 silencing on the proliferation, migration and invasion of $\mathrm{CC}$ cells.

Results: miR-145-5p was shown to be down-regulated in CC tissues and cells, while KLF5 was up-regulated. miR-145-5p could bind to the complementary sequence within the wild type KLF5 3'UTR rather than the mutant one. In addition, miR-145-5p could effectively down-regulate KLF5, in turn inhibiting the proliferation, migration and invasion of CC cells. Conclusion: miR-145-5p regulates the proliferation, migration and invasion of CC cells by targeting KLF5.

Keywords: cervical carcinoma, miR-145-5p, KLF5, proliferation, migration, invasion

\section{Introduction}

Cervical carcinoma (CC) is the second common cancer among women with higher morbidity and mortality. ${ }^{1,2}$ Almost $95 \% \mathrm{CC}$ is caused by persistent infection of human papillomavirus (HPV). ${ }^{3,4}$ Despite the pivotal role in the transformation of cervical epithelial cells, HPV infection is not enough to result in the malignant conversion and consequently lead to tumor occurrence. ${ }^{5}$ In the infected bodies, CC progression can be affected by various auxiliary factors, especially by some differentially expressed genes. 6,7 Therefore, knowing more about the abnormally expressed genes can help to find novel therapeutic methods for $\mathrm{CC}$ and improve patient's prognosis.

MicroRNAs (miRNAs) have been found to be abnormally expressed in a variety of cancer types, and they can catalyze mRNA cleavage or inhibit translation in the way of interacting with the complementary sequences of target mRNAs, thus affecting cancer progression. ${ }^{8-10} \mathrm{Wu}$ et al discovered that miR-144-3p was significantly down-regulated in $\mathrm{CC}$, and it inhibited cell proliferation, migration and invasion by targeting MAKP $6^{11}$. Li et al found that miR-93-5p played an inhibitory role in the 
proliferation and metastasis of CC cells, and it could suppress the development of HPV-positive CC by targeting BTG3. ${ }^{12}$ These studies suggest that the abnormal expression of miRNAs may affect the malignant progression of $\mathrm{CC}$. It has been reported that miR-145-5p is down-regulated in a variety of human cancers, such as breast cancer $^{13}$ and bladder cancer ${ }^{14}$, and plays an inhibitory role in tumor growth. In addition, miR-145 has been confirmed to have the potential serving as a candidate biomarker for human diagnosis. ${ }^{15}$ Sathyanarayanan et al also found that miR-145 inhibited the proliferation and metastasis of CC cells by targeting SIP1. ${ }^{16}$ Moreover, some bioinformatics studies have reported that the down-regulation of miR-145 is associated with poor prognosis of CC. ${ }^{17,18}$ Therefore, it is meaningful to study the potential mechanism of miR-145-5p in CC. Generally, miRNAs function in cancer via their target genes. KLF5, a member of Krüppel-like transcription factor (KLF) family, participates in a variety of biological processes, such as cell proliferation, differentiation, growth, etc. ${ }^{19,20}$ Studies have shown that high KLF5 can promote cell growth and cause phenotypic changes, and it has been considered to be a novel target for cancer treatment. ${ }^{21,22}$ These studies collectively suggest that miR-145-5p and KLF5 may affect the malignant progression of CC.

Currently, about $70 \%$ of CC cases have been prevented by HPV vaccines (HPV-16 and HPV-18). ${ }^{19}$ In this study, we used the bioinformatics technique and in vitro experiments to clarify the molecular mechanism of the miR-145-5p/KLF5 axis underlying the occurrence and development of $\mathrm{CC}$, and aimed to provide a theoretical support for the targeted therapy of CC.

\section{Materials and Methods}

\section{Bioinformatics Analysis}

Expression data of miRNAs and mRNAs of $\mathrm{CC}$ were accessed from TCGA database (https://cancergenome.nih. gov/). "edgeR" package was used to perform differential analysis $(|\operatorname{logFC}|>2$, Padj $<0.01)$ to obtain the differentially expressed miRNAs (DEmiRNAs) and mRNAs (DEmRNAs). Then survival analysis was conducted and confirmed miR$145-5 p$ to be our research object. TargetScan, miRDB and miRTarBase three databases were applied to predict the downstream target mRNAs of miR-145-5p, and a Venn diagram was made to find the potential target mRNA.

\section{Cell Culture and Transfection}

Human CC cell lines C33A (HTB-31), HT-3 (HTB-32), Hela (CCL-2) and normal cervical cell line HcerEpic
(AC340374) were cultured in the Dulbecco's Modified Eagle Medium (DMEM; 30-2002) containing 10\% fetal bovine serum (FBS), and maintained in a $37^{\circ} \mathrm{C}$ incubator containing $5 \% \mathrm{CO}_{2}$. These cells and culture mediums were purchased from the American Type Culture Collection (ATCC; Manassas, VA, USA).

$\mathrm{CC}$ cells in logarithmic phase were divided into negative control (NC) mimic, miR-145-5p mimic, siRNA (si)NC, si-KLF5, NC mimic + si-NC, miR-145-5p mimic + si-NC and miR-145-5p mimic + si-KLF5 groups. NC mimic, miR-145-5p mimic, si-NC and si-KLF5 (Ribo Bio; Guangzhou, China) were transiently transfected into cells using Lipofectamine ${ }^{\circledR} 2000$ reagent (Thermo Fisher Scientific, Inc., Waltham, USA), respectively, and then the cells were cultured in corresponding mediums with $5 \%$ $\mathrm{CO}_{2}$ at $37^{\circ} \mathrm{C}$. After $6 \mathrm{~h}$, the culture mediums were changed and the cells were continuously cultured for further 48 $\mathrm{h}$ for follow-up experiments.

\section{Real-Time Quantitative PCR (qRT-PCR)}

Total RNA was extracted using the TRIzol reagent (Invitrogen, Thermo Fisher Science, Inc.), and then reversely transcribed into cDNA by the SuperScript ${ }^{\circledR}$ II Reverse Transcriptase kit (Invitrogen, USA). qRT-PCR was performed using the SYBR $^{\circledR}$ Premix Ex Taq ${ }^{\mathrm{TM}}$ (Takara, Japan) under the following thermal cycling conditions: pre-denaturation at $95^{\circ} \mathrm{C}$ for $10 \mathrm{~min}, 40$ cycles at $95^{\circ} \mathrm{C}$ for $15 \mathrm{~s}, 60^{\circ} \mathrm{C}$ for $30 \mathrm{~s}$ and $72^{\circ} \mathrm{C}$ for $30 \mathrm{~s}$. U6 and GAPDH were applied as endogenous regulators. The relative expression levels of the target miRNA and mRNA were calculated using the $2^{-\Delta \Delta C t}$ method. The primers used were synthesized by the GeneCopoeia (Maryland, USA) and the sequences were as follows: miR145-5p (F: 5'-CTCACGGTCCAGTTCCCA-3'; R: 5'-ACC TCAAGAACAGTATTTCCAGG-3'); U6 (F: 5'-GCTTCGG CAGCACATATACTAAAAT-3'; R:5'-CGCTTCACGAATT TGCGTGTCAT-3'); KLF5 (F: 5'-ACACCAGACCGCAGC TCCA-3'; R: 5'-TCCATTGCTGCTGTCTGATTTGTAG-3'); GAPDH (F: 5'-ACAGTCAGCCGCATCTTCTT-3'; R: 5'ACGACCAAATCCGTTGACTC-3').The experiment was repeated 3 times independently.

\section{Western Blot}

Total proteins were isolated from cells with RIPA lysate buffer (P0013C, Beyotime, Jiangsu, China). BCA Protein Assay Kit (23227, Thermo Fisher Scientific) was used to determine the concentrations of the protein samples, and the samples were quantified according to different concentrations. Thereafter, the proteins were boiled for $10 \mathrm{~min}$ at 
$95{ }^{\circ} \mathrm{C}$ with $10 \mu \mathrm{L}$ of loading buffer (1610737, Haoran Biological Technology, Shanghai, China) and then separated by sodium dodecyl sulfate polyacrylamide gel electrophoresis (SDS-PAGE) at 100V. After electrophoresis, the proteins were transferred onto nitrocellulose membranes, which were sequentially blocked by $5 \% \mathrm{BSA} /$ TBST for $60 \mathrm{~min}$. Primary rabbit polyclonal antibodies KLF5 (ab137676, 1:2000, Abcam, Cambridge, USA) and GAPDH (ab181602, 1:1000, Abcam) were added onto the membranes for incubation overnight at $4^{\circ} \mathrm{C}$, followed by secondary antibody goat anti-rabbit IgG H\&L (ab216773, 1:1500, Abcam) at room temperature for $1 \mathrm{~h}$. The membranes were washed with TBST buffer for 3 times. An Enhanced Chemiluminescence (ECL) kit (ECL808-25, Biomiga, USA) was used for the visualization of protein bands, and the Image Pro Plus 6.0 (Media Cybernetics, USA) software was applied to analyze the relative protein levels. The experiment was repeated 3 times independently.

\section{Dual-Luciferase Reporter Gene Assay}

The amplified sequences of the wild type 3' UTR of KLF5 (WT-3'UTR) were cloned into the pmirGLO vectors (Promega Corp., WI, USA) for the construction of luciferase Wt-KLF5 vectors, and the mutant ones (luciferase Mut-KLF5) were synthesized by GenScript (Nanjing, China). miR-145-5p mimic and $\mathrm{NC}$ mimic were cotransfected with Wt-KLF5 or Mut-KLF5 into cells, respectively. After 48 h, Firefly and Renilla luciferase activities were measured using the Dual-Luciferase Reporter system (Promega) following the manufacturer's instructions. The fluorescence intensity was measured by a luminometer TD-20/20 (E5311, Promega). The Firefly luciferase activity was normalized to Renilla luciferase activity for each sample. The experiment was repeated 3 times independently.

\section{CCK-8}

Cell Counting Kit-8 (CCK-8, Beyotime, Shanghai, China) was used to assess $\mathrm{CC}$ cell proliferation. Cell suspension were seeded into 96-well plates at a density of $8 \times 10^{3}$ cells/well, and maintained in $5 \% \mathrm{CO}_{2}$ at $37{ }^{\circ} \mathrm{C}$. At 0,24 , 48,72 and $96 \mathrm{~h}, 10 \mu \mathrm{L}$ of CCK-8 reagent was added into each well for $4 \mathrm{~h}$ of incubation, respectively. Then, the absorbance values were measured at $450 \mathrm{~nm}$. The experiment was repeated in triplicate.

\section{Transwell Invasion Assay}

24-well Transwell inserts (BD, Biosciences, MD, USA) with $8 \mu \mathrm{m}$ in aperture were used to detect cell invasion. About $2 \times 10^{4}$ cells were added into the upper chambers that were pre-coated with Matrigel matrix (BD Bioscience), and RPMI-1640 medium containing 10\% FBS was filled into the lower chambers as an attractant. After incubation at $37^{\circ} \mathrm{C}$ for $24 \mathrm{~h}$, the cells that did not invade to the lower chambers were removed with cotton swabs, and the invaded cells were fixed and stained with $100 \%$ methanol and $0.1 \%$ crystal violet. Five fields of the view were randomly selected for cell count under a microscope. The experiment was repeated 3 times independently.

\section{Wound Healing Assay}

Wound healing assay was performed for the detection of cell migration. Cells were inoculated in 6-well plates and a scrape across the single layer was made with the tip of a $200 \mu \mathrm{L}$ pipette when the cell confluence reached $80 \%$. The wells were briefly washed twice to remove the separated cells. Then, the cells were grown with fresh mediums. The scratch area was photographed with an inverted microscope after $0 \mathrm{~h}$ and $24 \mathrm{~h}$, and the wound healing rate was calculated using the Image J. The experiment was repeated 3 times independently.

\section{Statistical Analysis}

The SPSS v.24.0 software (IBM Corp., NY, USA) was applied for statistical analysis, and the GraphPad Prism 6 software (GraphPad Software, Inc., CA, USA) was used for the completion of graphical demonstrations. All data were expressed as mean \pm standard deviation (SD). Student's $t$ test was used for analyzing the comparisons between two groups, and one-way analysis of variance (ANOVA) was applied for the comparisons among multiple groups. $P<0.05$ was considered statistically significant.

\section{Results}

\section{miR-I45-5p Is Down-Regulated in CC Tissues and Cells}

"edgeR" package was used to identify the DEmiRNAs and DEmRNAs in the TCGA-CESC dataset. As shown in Figure 1A, it was found that miR-145-5p was significantly down-regulated in cancer tissues. Survival analysis showed that low miR-145-5p was markedly correlated with prognosis of CC patients (Figure 1B). These results 
suggested that the down-regulation of miR-145-5p could lead to the tumor progression. Afterwards, miR-145-5p was further detected in human normal cervical cell line HcerEpic and human CC cell lines C33A, HT-3 and Hela by qRT-PCR (Figure 1C). It was found that miR-145-5p was remarkably decreased in cancer cases relative to the normal cell line, and the lowest expression was shown in Hela cells. Therefore, Hela cells were chosen for followup experiments.

\section{KLF5 Is Up-Regulated in CC Tissues and Cells}

In order to further understand the downstream regulatory mechanism of miR-145-5p in CC, we used miRDB, miRTarBase and TargetScan databases to predict the target
mRNAs of miR-145-5p. The Venn diagram (Figure 2A) was made to find the overlapping mRNAs, among which KLF5 was with the putative binding sites with miR-145$5 p$. Subsequently, KLF5 level was determined in the TCGA-CESC dataset, finding that KLF5 was significantly up-regulated in tumor tissues compared with normal tissues (Figure 2B). Meanwhile, qRT-PCR was conducted to test KLF5 in CC cells, and the results showed that KLF5 mRNA was significantly up-regulated in $\mathrm{CC}$ cell lines relative to the normal cell line (Figure 2C).

\section{KLF5 Is a Direct Target of miR-I45-5p in $\mathrm{CC}$}

As abovementioned, putative binding sites of miR-145-5p on KLF5 were predicted using the bioinformatics methods.
A

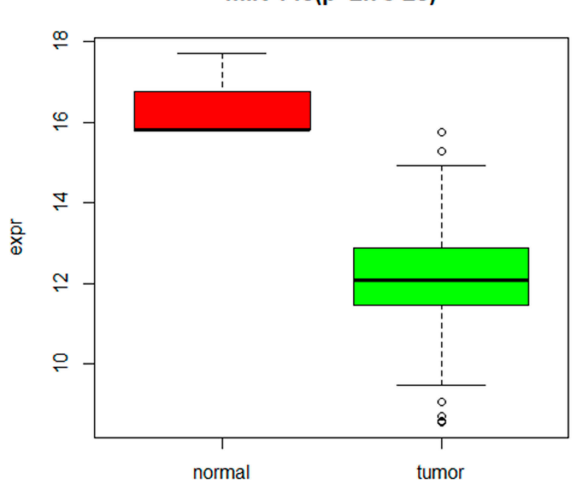

B

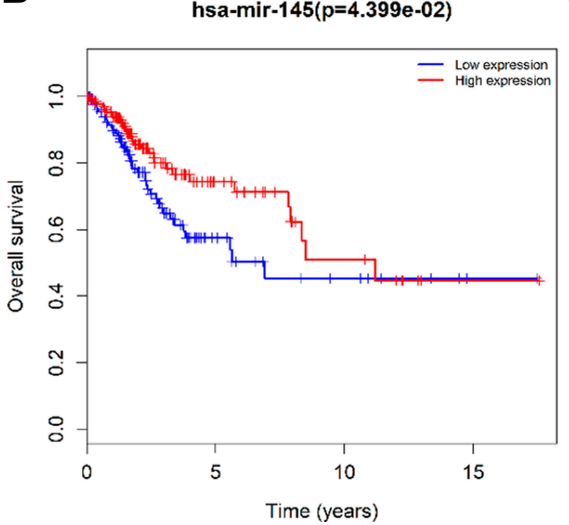

C

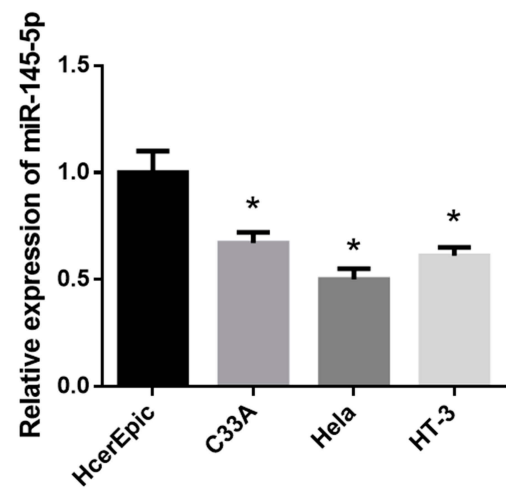

Figure I miR-145-5p is poorly expressed in CC tissues and cells. (A): Box diagram showed the levels of miR-145-5p in the TCGA-CESC dataset (green: tumor; red: normal); (B): Kaplan-Meier survival analysis of miR-I45 in the TCGA-CESC dataset. The abscissa represents time (years), and the coordinate represents survival rate (red: high expression; blue: low expression); $(\mathbf{C})$ : The relative expression of miR-I45-5p in human normal cervical cells and CC cells detected by $q R T-P C R$. $(* P<0$. 05).

A

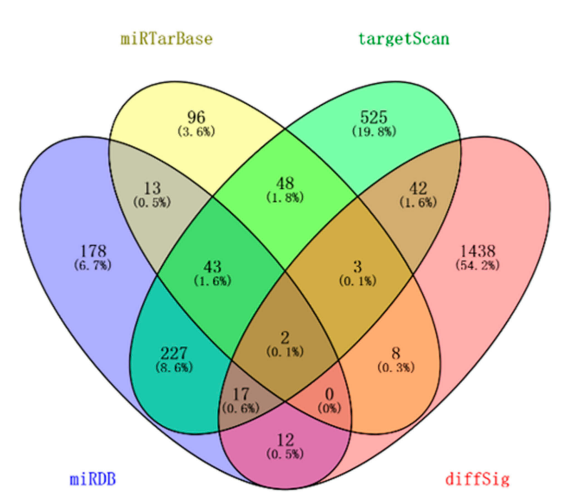

B

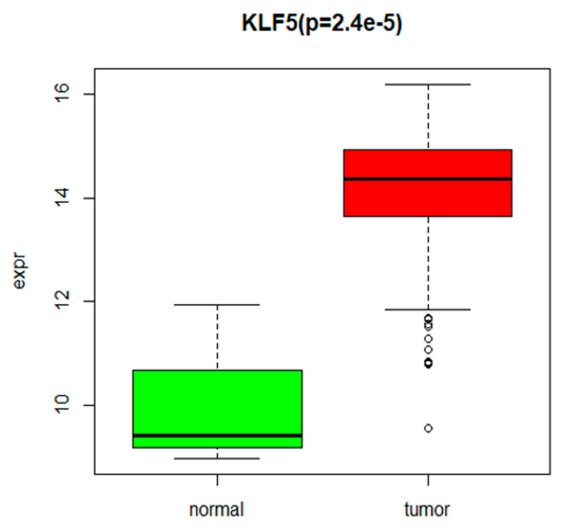

C

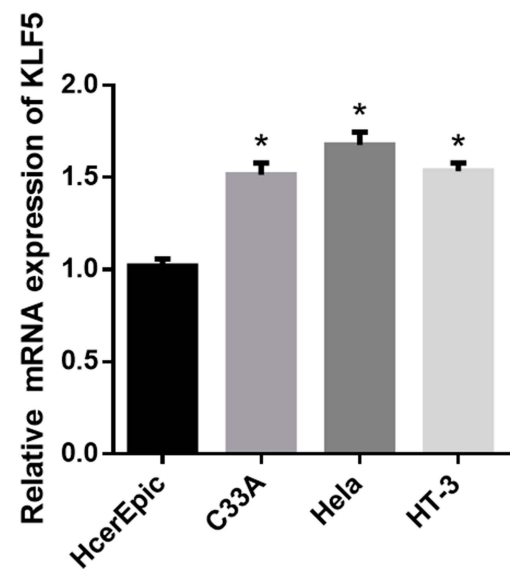

Figure 2 KLF5 is highly expressed in CC tissues and cells. (A): Venn diagram showed the overlapping mRNAs among the predictive target genes of miR-145-5p and DEmRNAs in the TCGA-CESC dataset; (B): Box diagram showed the levels of KLF5 in the TCGA-CESC dataset (green: normal; red: tumor); (C): The mRNA expression of KLF5 in human normal cervical cells and CC cells assessed by qRT-PCR. (*P<0. 05). 
A

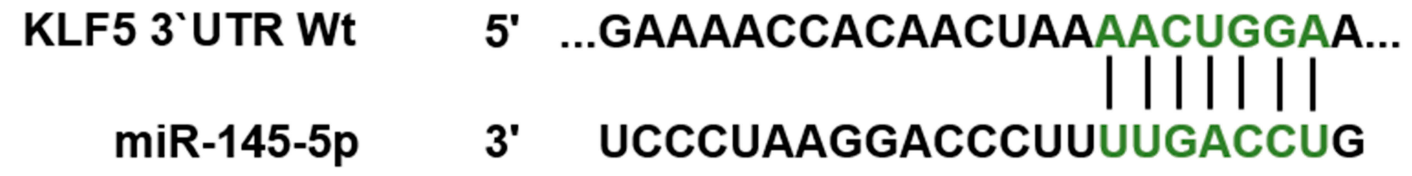

KLF5 3`UTR Mut 5' ...GAAAACCACAACUAACACGAUUA...

B

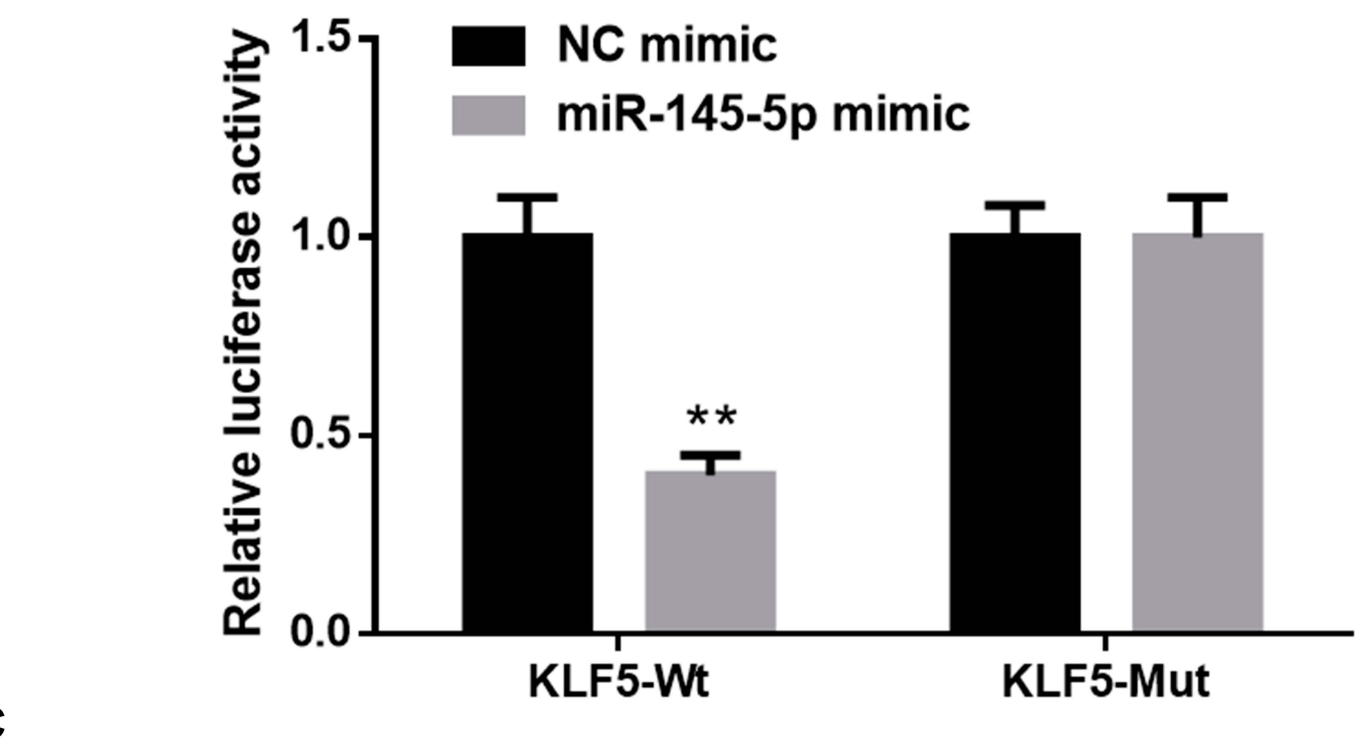

C

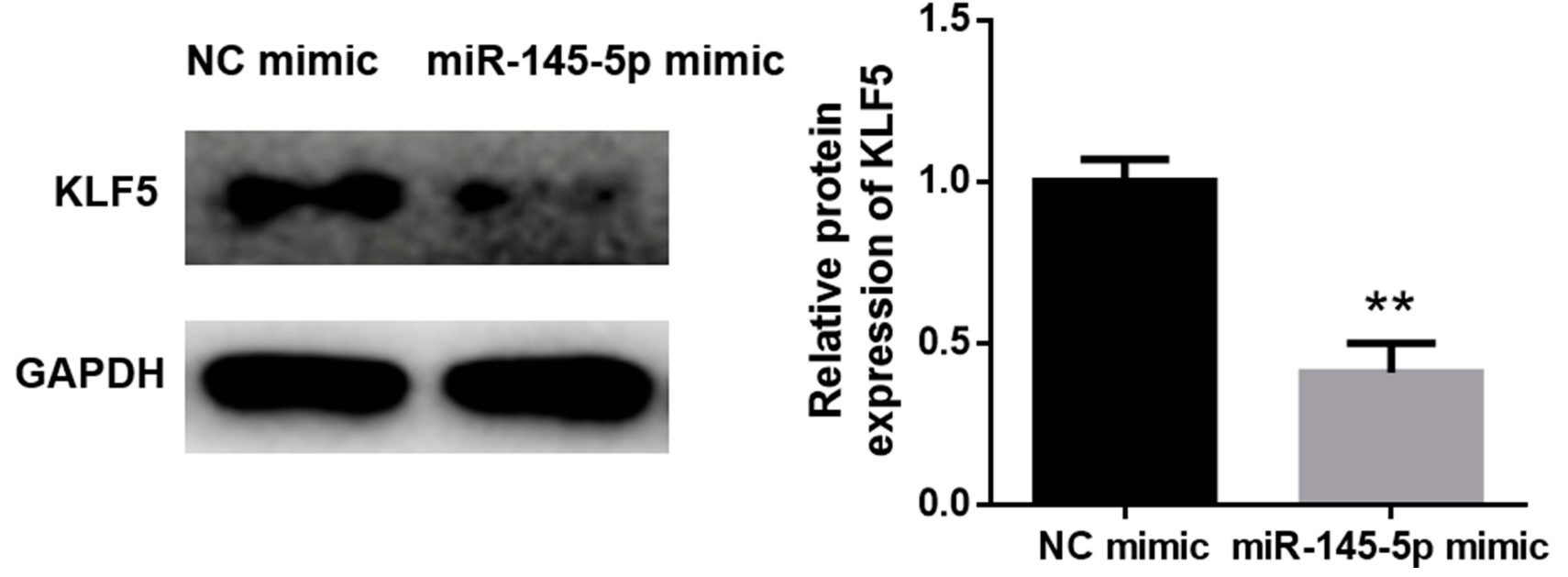

Figure 3 KLF5 is a direct target of miR-145-5p in CC. (A): The predicted binding sites of miR-I45-5p on KLF5; (B): Dual-luciferase reporter assay was conducted to verify the targeted relationship between miR-145-5p and KLF5; (C): The protein levels of KLF5 in different treatment groups. (**P < 0 . 0 I).

To clarify the specific relationship between miR-145-5p and KLF5, NC mimic and miR-145-5p mimic were transfected into cancer cells. A dual-luciferase reporter assay was performed for further verification (Figure 3A and B) and revealed that overexpressing miR-145-5p could significantly decrease the luciferase activity of the cells transfected with Wt-KLF5, while there was no significant difference observed in the cells with Mut-KLF5. In addition, the protein level of KLF5 in HeLa cells transfected with miR-145-5p mimic was also inhibited (Figure 3C). These results collectively suggested that KLF5 was a direct target gene of miR-145-5p in CC. 
A

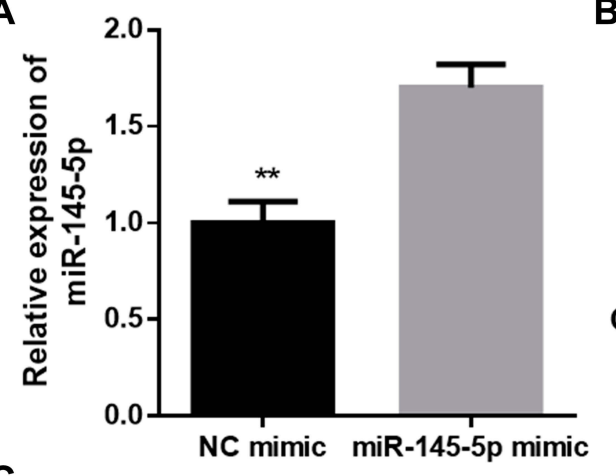

B

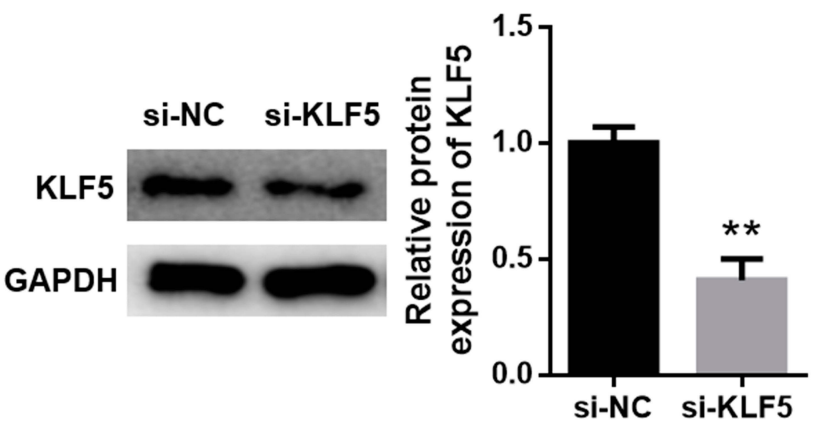

C

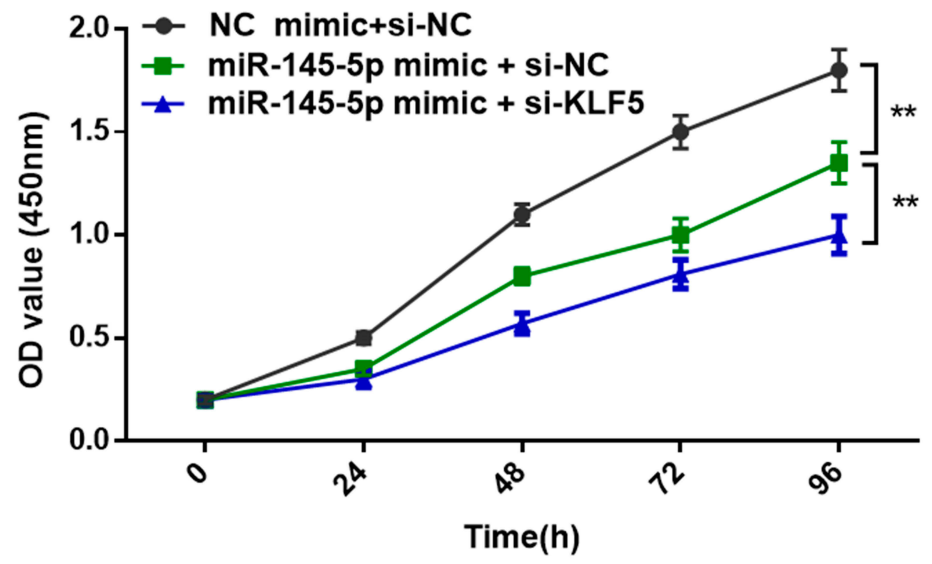

D

Oh
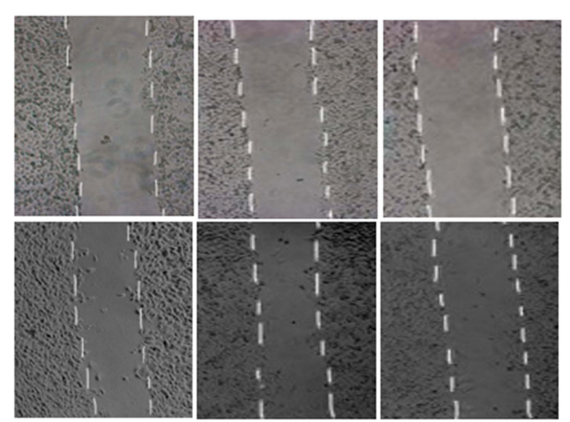

miR-145-5p mimic

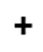

si-KLF5

E

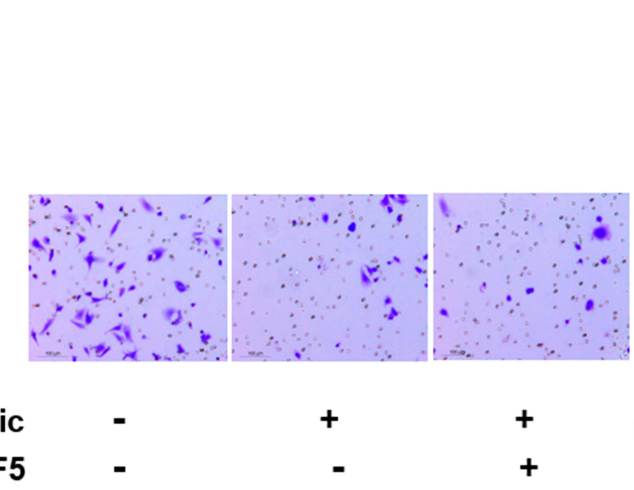

miR-145-5p mimic

si-KLF5

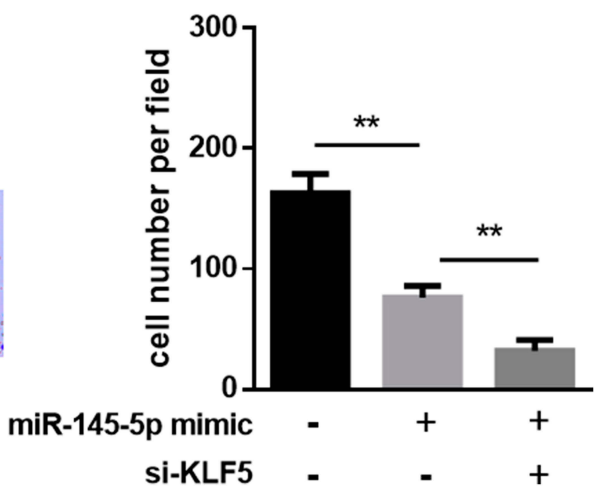

Figure 4 Effects of the miR-I45-5p/KLF5 axis on cell proliferation, migration and invasion in CC. (A), (B): qRT-PCR and Western blot were performed for detecting the transfection efficiency; (C): Cell proliferation assessment by CCK-8; (D), (E): Cell migration and invasion test via wound healing assay and Transwell invasion assay. $(* * P<0.01)$. 


\section{Effects of the miR-145-5p/KLF5 Axis on Hela Cell Proliferation, Migration and Invasion}

In the previous experiments, we verified that miR-145-5p could targeted regulate the expression of KLF5. In view of this, we further explored the role of the miR-145-5p/KLF5 regulatory axis in the malignant progression of CC. Hela cells were divided into three groups for in vitro experiments: $\mathrm{NC}$ mimic + si-NC, miR-145-5p mimic + si-NC and miR-145-5p mimic + si-KLF5 groups. qRT-PCR and Western blot were performed to determine the transfection efficiency, and found that miR-145-5p was significantly increased in the miR-145$5 \mathrm{p}$ mimic + si-NC group relative to the $\mathrm{NC}$ mimic + si-NC group, whereas KLF5 was remarkably decreased in the miR$145-5 p$ mimic + si-KLF5 group relative to the miR-145-5p mimic + si-NC group (Figure 4A and B). Subsequently, cell biological behaviors were assayed. CCK- 8 , transwell invasion and wound healing assays suggested that miR-145-5p overexpression played an inhibitory role in cell proliferation, invasion and migration abilities, and such effect could be enhanced when KLF5 was simultaneously silenced (Figure 4C-E). Taken together, these results indicated that miR-145-5p could inhibit the proliferation, migration and invasion abilities of CC cells by down-regulating the expression of KLF5.

\section{Discussion}

Although the development of the HPV vaccine has contributed to the reduction of the risk of $\mathrm{CC}$ in women, it is still very important to explore the molecular mechanisms underlying the malignant progression of $\mathrm{CC}^{23}$ Several studies have shown that miR-145 plays an anti-tumor role in a variety of human cancers. ${ }^{24,25}$ Sathyanarayanan et al found that miR-145 was down-regulated in CC, and the level of miR-145 showed an intimate correlation with prognosis. ${ }^{16,26}$ In the present study, miR-145-5p was found to be markedly decreased in $\mathrm{CC}$ tissues through the differential analysis on the miRNA expression data in the TCGA-CESC dataset. In addition, survival analysis showed that patients with high miR-145 had a better prognosis, which is in agreement with the previous studies. ${ }^{17,18}$ Moreover the expression of miR-145-5p in CC cell lines was also detected, and similar results were obtained.

At present, the direct target mRNAs of miR-145 in CC have been reported to be Fascin Actin-Bundling Protein 1 (FSCN1), ${ }^{27}$ SMAD-interacting protein 1 (SIP1), ${ }^{16}$ octamerbinding transcription factor 4 (OTC4 $)^{28}$ and myosin phosphatase targeting subunit 1 (MYTP1). ${ }^{29}$ In other words,
KLF5 has not been studied as a target of miR-145-5p in CC. Although previous studies have shown that KLF5 is closely related to the occurrence and development of CC, the underlying molecular mechanism has not been fully discussed. Zhang et al reported that miR-152 functioned on CC by inhibiting KLF5. ${ }^{30}$ Besides, they found that KLF5 was highly expressed in CC and the down-regulation of KLF5 could result in the inhibition of cell proliferation and cell cycle progression but showed no relevance to the cell metastasis. Moreover, Ma et al suggested that KLF5 promoted the proliferation and invasion of $\mathrm{CC}$ cells, which partly depended on the expression of TNFRSF11a. ${ }^{31}$ Similarly, our study confirmed that KLF5 was highly expressed in CC. Furthermore, the results of in vitro experiments showed that miR-145-5p could inhibit the proliferation, migration and invasion of CC cells by down-regulating KLF5, which has not been reported in previous studies. ${ }^{32,33}$

To sum up, our study found that overexpressing miR$145-5 p$ or silencing KLF5 could suppress the malignant progression of $\mathrm{CC}$, and revealed the targeted relationship between the two genes for the first time. The discovery of the miR-145-5p/KLF5 regulatory axis also provides a theoretical support for mining molecular targets for CC targeted therapy. However, this study also has some limitations. For example, our research on the potential mechanism of miR-145-5p in the occurrence and development of CC has only focused on the target mRNA of miR$145-5 p$, which prompts us to make an in-depth study. In further study, we will analyze the enriched pathways of KLF5 in CC and explore the potential molecular mechanism of the miR-145-5p/KLF5-related signaling pathways underlying the malignant progression of $\mathrm{CC}$.

\section{Disclosure}

The authors report no conflict of interest in this work.

\section{References}

1. Dizon DS, Mackay HJ, Thomas GM, et al. State of the science in cervical cancer: where we are today and where we need to go. Cancer. 2014;120(15):2282-2288. doi:10.1002/cncr.28722

2. Moore DH. Cervical cancer. Obstet Gynecol. 2006;107(5):1152-1161. doi:10.1097/01.AOG.0000215986.48590.79

3. Cervical cancer analysis reveals new mutations. Cancer Discov. 2017;7(4):344.

4. Forman D, de Martel C, Lacey CJ, et al. Global burden of human papillomavirus and related diseases. Vaccine. 2012;30(Suppl 5):F12F23. doi:10.1016/j.vaccine.2012.07.055

5. Burd EM. Human papillomavirus and cervical cancer. Clin Microbiol Rev. 2003;16(1):1-17. doi:10.1128/CMR.16.1.1-17.2003 
6. Schiffman M, Castle PE, Jeronimo J, et al. Human papillomavirus and cervical cancer. Lancet. 2007;370(9590):890-907. doi:10.1016/ S0140-6736(07)61416-0

7. Snijders PJ, Steenbergen RD, Heideman DA, et al. HPV-mediated cervical carcinogenesis: concepts and clinical implications. J Pathol. 2006;208(2):152-164. doi:10.1002/(ISSN)1096-9896

8. Berindan-Neagoe I, Monroig PDC, Pasculli B, et al. MicroRNAome genome: a treasure for cancer diagnosis and therapy. CA Cancer J Clin. 2014;64(5):311-336. doi:10.3322/caac.21244

9. Di Leva G, Garofalo M, Croce CM. MicroRNAs in cancer. Annu Rev Pathol. 2014;9(1):287-314. doi:10.1146/annurev-pathol-012513104715

10. Rupaimoole R, Slack FJ. MicroRNA therapeutics: towards a new era for the management of cancer and other diseases. Nat Rev Drug Discov. 2017;16(3):203-222. doi:10.1038/nrd.2016.246

11. Hu X, Miao J, Zhang M, et al. miRNA-103a-3p promotes human gastric cancer cell proliferation by targeting and suppressing ATF7 in vitro. $\mathrm{Mol}$ Cells. 2018;41(5):390-400. doi:10.14348/molcells.2018.2078

12. Liu J, Bian T, Feng J, et al. miR-335 inhibited cell proliferation of lung cancer cells by target Tra2ß. Cancer Sci. 2018;109(2):289-296. doi:10.1111/cas.13452

13. Wu J, Zhao Y, Li F, et al. MiR-144-3p: a novel tumor suppressor targeting MAPK6 in cervical cancer. J Physiol Biochem. 2019;75 (2):143-152. doi:10.1007/s13105-019-00681-9

14. Li J, Chu Z-P, Han H, et al. Suppression of miR-93-5p inhibits high-risk HPV-positive cervical cancer progression via targeting of BTG3. Hum Cell. 2019;32(2):160-171. doi:10.1007/s13577-01800225-1

15. Tang W, Zhang X, Tan W, et al. miR-145-5p suppresses breast cancer progression by inhibiting SOX2. J Surg Res. 2019;236:278-287. doi:10.1016/j.jss.2018.11.030

16. Sun M, Zhao W, Chen Z, et al. Circular RNA CEP128 promotes bladder cancer progression by regulating Mir-145-5p/Myd88 via MAPK signaling pathway. Int $J$ Cancer. 2019;145(8):2170-2181. doi:10.1002/ijc.v145.8

17. Wei H, Wen-Ming C, Jun-Bo J. Plasma miR-145 as a novel biomarker for the diagnosis and radiosensitivity prediction of human cervical cancer. J Int Med Res. 2017;45(3):1054-1060. doi:10.1177/ 0300060517709614

18. Sathyanarayanan A, Chandrasekaran KS, Karunagaran D. microRNA-145 modulates epithelial-mesenchymal transition and suppresses proliferation, migration and invasion by targeting SIP1 in human cervical cancer cells. Cell Oncol (Dordr). 2017;40 (2):119-131. doi:10.1007/s13402-016-0307-3

19. Huang L, Lin J-X, Yu Y-H, et al. Downregulation of six microRNAs is associated with advanced stage, lymph node metastasis and poor prognosis in small cell carcinoma of the cervix. PLoS One. 2012;7 (3):e33762. doi:10.1371/journal.pone.0033762
20. Li MY, Hu XX. Meta-analysis of microRNA expression profiling studies in human cervical cancer. Med Oncol. 2015;32(6):510. doi:10.1007/s12032-015-0510-5

21. Ghaleb AM, NANDAN MO, CHANCHEVALAP S, et al. Krüppellike factors 4 and 5: the yin and yang regulators of cellular proliferation. Cell Res. 2005;15(2):92-96. doi:10.1038/sj.cr.7290271

22. Shi M, Du L, Liu D, et al. Glucocorticoid regulation of a novel HPV-E6-p53-miR-145 pathway modulates invasion and therapy resistance of cervical cancer cells. J Pathol. 2012;228(2):148-157. doi:10.1002/path.v228.2

23. Gao Y, Ding Y, Chen H, et al. Targeting Krüppel-like factor 5 (KLF5) for cancer therapy. Curr Top Med Chem. 2015;15(8):699-713. doi:10.2174/1568026615666150302105052

24. Shindo T, Manabe I, Fukushima Y, et al. Krüppel-like zinc-finger transcription factor KLF5/BTEB2 is a target for angiotensin II signaling and an essential regulator of cardiovascular remodeling. Nat Med. 2002;8(8):856-863. doi:10.1038/nm738

25. Joura EA, Giuliano AR, Iversen O-E, et al. A 9-valent HPV vaccine against infection and intraepithelial neoplasia in women. $N \mathrm{Engl}$ $J$ Med. 2015;372(8):711-723. doi:10.1056/NEJMoa1405044

26. Boufraqech M, Zhang L, Jain M, et al. miR-145 suppresses thyroid cancer growth and metastasis and targets AKT3. Endocr Relat Cancer. 2014;21(4):517-531. doi:10.1530/ERC-14-0077

27. Lei C, Du F, Sun L, et al. miR-143 and miR-145 inhibit gastric cancer cell migration and metastasis by suppressing MYO6. Cell Death Dis. 2017;8(10):e3101. doi:10.1038/cddis.2017.493

28. Ye C, Sun N-X, Ma Y, et al. MicroRNA-145 contributes to enhancing radiosensitivity of cervical cancer cells. FEBS Lett. 2015;589 (6):702-709. doi:10.1016/j.febslet.2015.01.037

29. Ma L, Li LL. miR-145 contributes to the progression of cervical carcinoma by directly regulating FSCN1. Cell Transplant. 2019;28 (9-10):1299-1305. doi:10.1177/0963689719861063

30. Yan S, Li X, Jin Q, et al. MicroRNA-145 sensitizes cervical cancer cells to low-dose irradiation by downregulating OCT4 expression. Exp Ther Med. 2016;12(5):3130-3136. doi:10.3892/etm.2016.3731

31. González-Torres A, Bañuelos-Villegas EG, Martínez-Acuña N, et al. MYPT1 is targeted by miR-145 inhibiting viability, migration and invasion in 2D and 3D HeLa cultures. Biochem Biophys Res Commun. 2018;507(1-4):348-354. doi:10.1016/j.bbrc.2018.11.039

32. Zhang H, Lu Y, Wang S, et al. MicroRNA-152 acts as a tumor suppressor microRNA by inhibiting Krüppel-like factor 5 in human cervical cancer. Oncol Res. 2019;27(3):335-340. doi:10.3727/ 096504018X15252202178408

33. Ma D, Chang L-Y, Zhao S, et al. KLF5 promotes cervical cancer proliferation, migration and invasion in a manner partly dependent on TNFRSF11a expression. Sci Rep. 2017;7(1):15683. doi:10.1038/ s41598-017-15979-1
OncoTargets and Therapy

\section{Publish your work in this journal}

OncoTargets and Therapy is an international, peer-reviewed, open access journal focusing on the pathological basis of all cancers, potential targets for therapy and treatment protocols employed to improve the management of cancer patients. The journal also focuses on the impact of management programs and new therapeutic agents and protocols on patient perspectives such as quality of life, adherence and satisfaction. The manuscript management system is completely online and includes a very quick and fair peer-review system, which is all easy to use. Visit http://www.dovepress.com/ testimonials.php to read real quotes from published authors. 